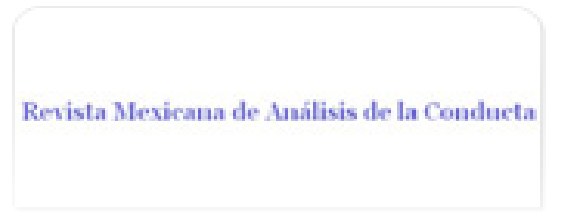

Revista Mexicana de Análisis de la Conducta ISSN: 0185-4534

editora@rmac-mx.org

Sociedad Mexicana de Análisis de la Conducta México

González Beltrán, Luis Fernando; Santoyo Velasco, Carlos Cooperación en dilemas de bienes públicos: el tamaño del grupo como señal de aportación Revista Mexicana de Análisis de la Conducta, vol. 33, núm. 2, 2007, pp. 205-224

Sociedad Mexicana de Análisis de la Conducta Guadalajara, México

Disponible en: http://www.redalyc.org/articulo.oa?id=59333206

- Cómo citar el artículo

- Número completo

- Más información del artículo

- Página de la revista en redalyc.org

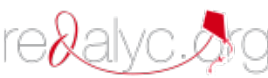

Sistema de Información Científica Red de Revistas Científicas de América Latina, el Caribe, España y Portugal Proyecto académico sin fines de lucro, desarrollado bajo la iniciativa de acceso abierto 


\title{
COOPERACIÓN EN DILEMAS DE BIENES PÚBLICOS: EL TAMAÑO DEL GRUPO COMO SEÑAL DE APORTACIÓN ${ }^{1}$
}

\author{
COOPERATION IN PUBLIC GOODS DILEMAS: \\ GROUP SIZE AS CONTRIBUTION SIGNAL \\ LUIS FERNANDO GONZÁLEZ BELTRÁN ${ }^{2}$ \\ Y CARLOS SANTOYO VELASCO ${ }^{3}$ \\ ${ }^{2}$ FES IZTACALA, Y ${ }^{3}$ FACULTAD DE PSICOLOGÍA, UNIVERSIDAD \\ NACIONAL AUTÓNOMA DE MÉXICO
}

\begin{abstract}
RESUMEN
Bajo una situación de "ahorro" e "inversión", 26 estudiantes de licenciatura se enfrentaron a un dilema de bienes públicos, donde la interacción entre los participantes se simuló por computadora, mientras las instrucciones indicaban que se efectuaba con los presentes en el aula. La tarea experimental se condujo en una sesión de 45 ensayos y consistió de problemas en los que se decidía cuanto aportar al grupo, de 5 puntos disponibles por ensayo. A fin de probar el efecto de señalización de la variable número de jugadores, se simuló un grupo de alta contribución $(80 \%)$, con un punto de provisión fijo en 24 unidades. Se mostró un efecto significativo del número de jugadores, donde se invirtió más en la condición de menos jugadores. El orden en que los participantes transitaron por las fases da cuenta del efecto del número de jugadores. Los resultados apoyan la hipótesis de cooperación condicional. Se abordan dos dimensiones de la conducta de los individuos: su ajuste a la aportación de su grupo de referencia y cómo los participantes intentan ganar control de la interacción. Se discuten las diferencias observadas y los factores que permiten el efecto del número de jugadores.
\end{abstract}

1. El presente es parte de la tesis doctoral del primer autor bajo la dirección del segundo, quienes agradecen el apoyo de DGAPA, UNAM al proyecto IN304405 y del CONACYT al proyecto 057327. Toda correspondencia dirigirla al primer autor: Av. Chihuahua 39 casa 27, Jardines de Atizapán, Atizapán de Zaragoza, C.P. 52978, México (correo electrónico: luisfgb@servidor.unam.mx). 
Palabras clave: cooperación condicional, bienes públicos, punto de provisión, tamaño de grupo, estudiantes.

\begin{abstract}
In a simulated public goods dilemma, framed as "saving" and "invest" games, 26 undergraduate students were instructed to play themselves, but in fact they interacted with a computer. Each subject participated once in a series of 45 independent single-trial dilemmas. On each trial participants received an endowment of five points to decide whether or not to contribute it for the provision of a public good. In order to evaluate the effects of group size to determine how much subjects contribute, they received information indicating that their group was relatively cooperative $(80 \%)$ with a provision point of 24 units. A significant effect of group size was found, with participants in the small group condition emitting more cooperative responses. Findings suggest that the effect depends on the particular sequence of experimental phases. Results lend some support to the conditional cooperation explanation. Our central focus was on the two dimensions of response available to subjects: adjustments in their individual contribution, and willingness to gain control of the interchange. The observed differences and the regulation factors of group size are discussed.
\end{abstract}

Keywords: social interaction, conditional cooperation, provision of steplevel public goods, group size, students.

Una "trampa social" es una situación de elección en la que dos o más individuos, al comportarse de manera egoísta, buscando ganancias a corto plazo, obtienen resultados desfavorables tanto colectiva como individualmente, en el mediano o largo plazo (Brechner, 1977; Platt, 1973). Las trampas sociales se han modelado como juegos experimentales, que consisten en interacciones estratégicas, situaciones en las que las consecuencias de las acciones de cada individuo dependen de las acciones tomadas por los otros, y esta interdependencia mutua es reconocida por los involucrados (Bowles, 2004).

Estos juegos pueden programarse para dos contrincantes, como el caso del "dilema del prisionero", pero las situaciones más realistas incluyen más jugadores que compiten por un recurso o bien común. En el arreglo típico del "dilema de bienes públicos", a cada uno de $\mathbf{n}$ jugadores se le dota de una cantidad $\mathbf{x}$ de fichas, que deben distribuirse entre dos destinos: uno privado y otro público. Por ejemplo a $\mathbf{4}$ jugadores se les dota de $\mathbf{5}$ fichas, y se les explican las dos opciones: a) pueden "retirar" sus fichas y guardarlas en su cuenta personal, o b) pueden "invertirlas" en un "negocio grupal", que con 
cierta probabilidad, puede reportar ganancias a todo el grupo. Esta situación se programa de forma que cada ficha en la cuenta personal represente una mayor ganancia (r) que cada ficha depositada en la cuenta pública (k), generando un sesgo hacia la individualidad (ya que $\mathbf{r}>\mathbf{k}$ ). Es decir, cada ficha que no se decide invertir es una ficha "ganada" $(\mathbf{r}=1)$, pero cada ficha que se destina al bien público solo significa la mitad de una ficha "ganada" para el sujeto que la aportó $(\mathbf{k}=1 / 2)$. En términos formales se tiene la ecuación:

$$
\boldsymbol{\Pi}=\mathbf{r} \mathbf{y}_{\mathbf{i}}+\mathbf{k} \sum \mathbf{x}_{\mathbf{i}} \quad(\text { Ecuación 1) }
$$

Donde $\boldsymbol{\Pi}$ es el total que recibe un jugador, que se compone de $\mathbf{y}_{\mathrm{i}}$ o las fichas que no invirtió (i es el número del jugador), más $\sum \mathbf{x}_{\mathbf{i}}$ (i va desde $\mathbf{1}$ hasta $\mathbf{n}$ ) o las fichas invertidas por todos los jugadores; cada sumando multiplicado por su factor de ponderación, $\mathbf{r}$ y $\mathbf{k}$.

En este ejemplo suponga que solo uno de los 4 jugadores decide aportar al bien público. Cada jugador que decide no aportar, de inicio se queda con sus 5 fichas (que no se devalúan puesto que el factor $r$ es de 1), más las que pudiera recibir como bien público por las aportaciones de los demás. El jugador que aporta sus 5 fichas al bien público, genera ganancias de 5 fichas más, pero el bien público de 10 fichas se reparte entre los 4 jugadores. El jugador que aporta termina con solo 2.5 fichas, habiendo iniciado con 5 , (al multiplicarse por el factor $\mathbf{k}$ ) y los tres jugadores que no aportaron terminan con 7.5 fichas. Si más jugadores aportaran, obtendrían mayores utilidades dado que la ganancia por ficha se multiplica por el número de jugadores que aportaron. En el caso de mayor cooperación grupal, en que los 4 jugadores aportan las 5 fichas, suman 20 fichas invertidas, las ganancias son otras 20 fichas, y el total de 40, al repartirse entre 4, da un total de 10 fichas a cada jugador. En el caso de nula cooperación, nadie aporta y el bien público no se puede crear, nadie gana más que sus 5 fichas de inicio.

En el juego estándar, se especifica que las decisiones deben tomarse en privado, que deben ser del tipo todo o nada (se aporta o no se aporta) y se debe hacer del conocimiento general cuanto se acumuló y cuanto recibe cada jugador. La estructura de estas situaciones se caracteriza porque el valor de cada curso de acción depende de cuantos lo usen (Kollock, 1998). De esta forma, la opción más rentable es no aportar, y aprovecharse del total que conjuntan los demás (con 4 jugadores y tres aportando, el que no aporta termina con 12.5 fichas contra 7.5 de los demás). Esta decisión se espera desde que la ganancia por ficha del bien público (r) es mayor que la ganancia por ficha del bien privado (k). Sin embargo, esta opción deja de ser la mejor conforme más individuos la adoptan, puesto que así dejan de generarse ganancias. De esta manera, se ha postulado que en el dilema de bienes públicos, solo interesan los valores de $\mathbf{r}$ y $\mathbf{k}$, en la ecuación 1. Pero en este trabajo 
se postula la existencia de otras variables implicadas que contribuirían a la comprensión del dilema.

La teoría económica ignora deliberadamente otras variables que no aparecen en la Ecuación 1, apostando por una estrategia individualista, ya que postula que un individuo "racional" toma elecciones que en todo momento deberán corresponder con las que con mayor probabilidad maximizarán su beneficio personal (Resnik, 1998). Al proponer que el comportamiento se debe solo a ese interés egoísta, se deja sin explicar porqué algunos individuos deciden aportar. De hecho, el hallazgo principal en bienes públicos es que en juegos de un único ensayo, cerca de la mitad de los participantes aportan (Ledyard, 1995). Para mantener a salvo la "racionalidad" de quienes aportan, se llegó a postular como explicación que los individuos no comprenden bien la tarea, y por tanto algunos deciden aportar (Kesser, 2000). Esta hipótesis se mantuvo gracias al hallazgo del patrón temporal cuando el juego de bienes públicos se conforma por múltiples ensayos: contribuciones cercanas al $50 \%$ en los ensayos iniciales, que declinan sustancialmente con el paso de los ensayos, prácticamente desapareciendo después de 10 ensayos (Ledyard, 1995). Si se piensa que los participantes comprenden tarde la tarea, se esperaría que después de unos cuantos ensayos, se dejará de cooperar, pero Andreoni (1988) mostró un efecto de restablecimiento: si se presenta, al terminar el primer juego, un segundo juego de bienes públicos, los individuos inician el nuevo juego con altos niveles de contribución, similares a los niveles iniciales del primero. Lo mismo sucede con un tercer juego, lo que invalida la explicación de que las aportaciones iniciales representan "errores" de los participantes.

En contraste, se han buscado otras formas de explicar las contribuciones. Para Axelrod (1984) el mecanismo que lo explica es la reciprocidad: así, la cooperación y el egoísmo son recíprocas: se coopera con los cooperativos y se traiciona a los egoístas. Esta definición intenta explicar el caso de juegos diádicos, como el dilema del prisionero, pero para el caso de bienes públicos, que es un juego de actores múltiples, se re-elaboró en otros términos. Así, Camerer y Fehr (2004) señalan: "reciprocidad significa que la gente está dispuesta a recompensar las acciones amigables y a castigar las acciones hostiles, aunque la recompensa o el castigo cause una reducción neta en el pago de quien recompensa o castiga" (subrayado en el original, p.7). Sin embargo, la opción de castigar no está disponible en todos los casos de bienes públicos, aunque los individuos pueden optar por la reciprocidad, ahora definida en términos de cooperación condicional: la conducta de los participantes en el juego de bienes públicos está orientada hacia la conducta promedio de los otros miembros del grupo en los ensayos previos (Kesser, 2000). Esta autora formaliza la reciprocidad en una regla de decisión cualitativa: si un sujeto intenta cambiar su decisión de un ensayo al siguiente, lo 
más probable es que cambie en la dirección del nivel de aportación de los otros miembros del grupo en el ensayo previo. Esto significa un incremento en la contribución si se está abajo del promedio, y un decremento si se está arriba. Los datos parecen seguir esta regla, y los "egoístas" no la violan en tanto que no cambian sus decisiones de un ensayo al siguiente, sino hasta que algo cambia, como la posibilidad de castigar la no-colaboración. Al mismo tiempo esta regla podría explicar la continua caída en la colaboración del experimento típico de esta forma: los participantes que contribuyen más que otros tienden a decrementar su contribución.

En el experimento de Fischbacher, Gachter y Fehr (2001), se evaluó la posibilidad de la cooperación condicional, aunque sin someter a los individuos al dilema, sino con un cuestionario, preguntando que tanto contribuirían al bien público si los demás aportaran tal o cual cantidad, en un único ensayo. La contribución promedio de los participantes que contestaron el cuestionario fue claramente creciente a la contribución promedio del grupo. Es decir, en promedio, los sujetos mostraron cooperación condicional. Lo que resalta son los patrones encontrados en el ámbito individual: cerca del $30 \%$ de los participantes optaron por la inversión privada a cualquier nivel de cooperación del grupo. Aparte de los "egoístas", están los cooperadores condicionales, un $50 \%$ del total, que cooperan más si el grupo coopera más. Un último patrón lo representa las contribuciones de un $14 \%$ de los sujetos, quienes ante contribuciones promedio y medianas, contribuyen como cooperadores condicionales, aumentando conforme lo hace el grupo, pero cuando llegan a un punto medio, conforme aumentan las contribuciones grupales, disminuyen sus contribuciones, hasta convertirse en egoístas ante grandes contribuciones del grupo.

Estos resultados muestran diferencias individuales en la aportación en bienes públicos. Para obtener patrones individuales, los autores requirieron cambiar de la decisión de todo o nada, a la de cuánto aportar en cada turno. Dicha decisión dependía de lo que se postulaba que aportaría el grupo, apoyando la hipótesis de la cooperación condicional, pero sin someterla a una prueba directa solicitando la decisión en un juego experimental. La razón para ello, es que el arreglo de bienes públicos, como todas las trampas sociales, tiene como característica definitoria su interdependencia, es decir la respuesta de uno de los jugadores afecta a sus propios resultados y a los resultados de los demás, lo que hacía más factible la pregunta directa a las distintas aportaciones grupales. Para evaluar la decisión del individuo como parte del grupo es necesario producir la toma de decisiones en un juego experimental. Aunque la pregunta directa es muy práctica, supone un individuo que sabe las aportaciones de los demás, antes de tomar su decisión. En este experimento se prefirió optar por producir una situación de bienes públicos manipulando directamente la cantidad que aportaría el grupo. Al hacerlo así, 
se simula la interdependencia con sujetos virtuales. Cada individuo tendría que decidir su aportación al bien público en un grupo simulado por computadora, aunque en una situación donde físicamente se encuentran todos los participantes experimentales, y con unas instrucciones que aseguran que entre ellos se conforma el grupo. Las razones para ello se justifican a continuación.

Para la hipótesis de cooperación condicional, la variable importante es la contribución del grupo: el individuo es sensible a lo que aportan los demás. Esto es de esperarse, dado que se ha demostrado que, en ocasiones, la gente tiene muy presente los resultados de quienes los rodean, al punto de compararse con los demás en términos de las opciones, ganancias, esfuerzos y tiempo dedicado (Santoyo, 2001), comparaciones que también deben evaluarse como factores en bienes públicos. La contribución grupal sirve como "señal" de qué dirección debe tomar un cambio, una vez que el sujeto decide cambiar, pero es posible que haya más variables que determinen el cambio, sobretodo si no se ha llegado a un punto de equilibrio.

Se supone que antes de desarrollar una estrategia, los individuos muestrean diferentes opciones, y se guían por reglas, o por otras variables que señalen la opción más adecuada, variables que deben someterse a control experimental. Adicionalmente, se ha reconocido la importancia de saber lo que hacen los jugadores en situaciones fuera de equilibrio; en la dinámica de las primeras fases del dilema, el concepto de aprendizaje es un complemento al concepto de solución al juego (Bowles, 2004).

Esta solución ya se ha ensayado para examinar la aportación en el dilema del prisionero para 5 jugadores, simulando una situación aleatoria, o una estrategia recíproca de los jugadores virtuales (Yi y Rachlin, 2004). Los participantes aportaron en mayor medida cuando los jugadores virtuales usaban la estrategia recíproca que cuando jugaron al azar. En el primer caso, aún la estrategia virtual dependía enteramente de los participantes reales, si aportaban, el grupo también lo hacía, si caían en la trampa de no aportar, el grupo dejaba de aportar. En ambas situaciones se producía un círculo, virtuoso o vicioso, que difícilmente rompían los participantes reales.

En el presente experimento, se utilizaron jugadores virtuales totalmente independientes de los participantes reales, a fin de probar el supuesto de la posible influencia de variables de señalización, para tomar la decisión de cuanto aportar en una situación de bienes públicos, adicionales a los valores $\mathbf{r}$ y $\mathbf{k}$ postulados en la Ecuación 1. De acuerdo a la propuesta de la cooperación condicional, la variable más importante a tener en cuenta es la contribución grupal. Una segunda variable sería el punto de provisión, mínimo de contribución debajo del cual toda aportación no solo no generaría intereses (bien público), sino que se perdería. En un experimento por Isaac, Schmitz, y Walker (1989), se compararon las aportaciones con o sin punto de pro- 
visión, conforme crecía el punto de provisión, las contribuciones del grupo incrementaban. Cuando el punto de provisión era del $100 \%$ de las fichas, las contribuciones tendían a elevarse aún más, aunque en muchos grupos no se alcanzaba el total. El mínimo de contribución grupal, o punto de provisión, podría señalar un nivel conjunto esperado de contribución a la cuenta del grupo y ayudaría a inducir expectativas comunes de contribuciones sustanciales a dicha cuenta. Con iguales dotaciones, la señal implicada sería que cada sujeto debería contribuir un enésimo del punto mínimo anunciado. Por tanto el número de jugadores es la tercera variable. Al manipularse el número de jugadores se han encontrado resultados contradictorios en la cooperación (Komorita y Lapworth, 1982; Isaac y Walter, 1988). Finalmente, una variable adicional que se tendría que evaluar, es la experiencia en el juego.

La literatura reporta muchas investigaciones donde se manipulan una o más de las variables mencionadas (véanse las revisiones de Ledyard, 1995; Bowles, 2004; Camerer y Fehr, 2004), con excepción de la contribución del grupo, que como se ha subrayado es una variable atributiva del grupo que se estudia. Esta variable podría explicar el patrón temporal de las aportaciones, pero es una variable que depende del grupo, y que puede cambiar momento a momento. Si los individuos son sensibles a la contribución del grupo, su ejecución cambiará si cambian de grupo. En el transcurso de la sesión el individuo también cambia su elección conforme el grupo cambia sus aportaciones. Dada esta dinámica, las manipulaciones experimentales de otras variables se confunden con las variaciones en el patrón temporal de la aportación grupal. De esta forma no es de extrañar que el número de jugadores, por ejemplo, en ocasiones produzca mayor cooperación y en otras, menor cooperación, o a veces no sea factor. Lo mismo podría decirse del papel que juegan otras variables. Por ello, es necesario mantener constante la aportación grupal a fin de evaluar correctamente el efecto de otras variables de señalización implicadas en el dilema de bienes públicos.

Si la hipótesis de la cooperación condicional es correcta, la decisión de cuánto aportar debería estar en relación directa con la contribución simulada del grupo, y si esta contribución se mantiene, el patrón temporal de la aportación individual no debería decrementar después de unos 10 ensayos. Si la contribución grupal se mantiene relativamente estable, se espera que este patrón fuese una excelente línea base para poder manipular otras variables, como es el caso del número de jugadores, que ha producido resultados irreconciliables. A fin de hacer más contundente su papel como factor de señalización, en el presente trabajo, se utilizó un punto de provisión, o mínimo de contribución grupal, fijo en todo el experimento.

Así, se expuso a los participantes a un valor extremo de contribución del grupo ( $80 \%$ del total), que simulaba un grupo de alta aportación al "negocio" grupal. En este contexto, se probaron tres valores de tamaño del grupo, 6, 8 o 
12 jugadores, en una situación de bienes públicos con punto de provisión fijo de 24 unidades, que representa el 80, 60 o 40\% del total, respectivamente, para cada valor de tamaño de grupo.

\section{MÉTODO}

PARTICIPANTES. Fueron 26 estudiantes de primer o tercer semestre de la licenciatura en Psicología, de una institución pública. Se condujeron dos sesiones mezclando individuos de diferentes semestres o grupos escolares. Todos participaron en solo una sesión, y al final de cada una se premiaron los 3 puntajes de ganancias más altos, de acuerdo a su sesión, otorgando $\$ 200$ al primer lugar, $\$ 150$ al segundo y $\$ 100$ al tercer lugar. Esto se hizo con el propósito de que los participantes estuvieran motivados, y que desarrollaran un comportamiento estratégico, o incluso que consideraran atractiva la estrategia de no aportar.

SITUACIÓN EXPERIMENTAL. Las sesiones se llevaron a cabo en un aula de cómputo, que contaba con 15 computadoras en 4 filas. Dado que el aula solo se abría por fuera, las sesiones se tuvieron que llevar a cabo con la puerta abierta, pero como se encuentra en un edificio de poco tránsito no representó un serio problema de distracción o de ruido. Cada sujeto se sentó frente a una computadora, y la interacción con su grupo se simulaba con la retroalimentación correspondiente a cada una de las condiciones experimentales, con un programa elaborado en Visual Basic 6.0 para Windows.

PROCEDIMIENTO. Se utilizó un diseño de medidas repetidas con un factor (número de jugadores) con 3 niveles (6, 8 y 12) en una sola sesión. Esta consistió de 45 ensayos donde cada uno de los participantes pasa por las tres fases, una para cada valor de número de jugadores. Cada fase consistió en 15 ensayos, y el orden de las fases (seis secuencias en total) se determinó aleatoriamente.

Se llevó a los estudiantes al aula y se les asignó una computadora, diciéndoles que participarían en un estudio sobre toma de decisiones grupales. El experimentador (el primer autor) dio una orientación general sobre el programa de cómputo y les dijo que las instrucciones tendrían que leerlas en pantalla, y que se encontrarían interactuando con algunos de los presentes, aunque la retroalimentación de la "aportación grupal", y las "ganancias grupales", la dio la computadora en términos de las diferentes condiciones experimentales. La retroalimentación de la ejecución propia, fue real.

La tarea experimental consistió de una simulación de problemas de bienes públicos con punto de provisión. Las instrucciones comprendían tres pantallas de texto que los participantes leían avanzando o retrocediendo a 
su ritmo ${ }^{2}$. En resumen, las instrucciones señalaron que el objetivo del juego era "acumular la mayor cantidad de puntos en la sesión", mediante una apropiada toma de decisiones, para obtener los premios disponibles. La tarea se describió como una situación de "invertir" en la que cada miembro del grupo debe decidir individualmente y en privado si mantiene su capital o lo invierte. Cada jugador debió elegir entre 0 y 5 puntos, para invertir en el "negocio común", lo que restara, se acumulaba en su "cuenta personal". El punto de provisión se definió de la siguiente manera: Si el número de inversionistas alcanzan o exceden cierto valor crítico, la "cuota de inversión", entonces cada miembro del grupo ya sea que haya invertido o no, recibiría el pago de "capital más intereses". Si no se alcanzaba la cuota de inversión, ningún miembro del grupo recibiría "ganancias".

Los participantes leyeron las instrucciones, y realizaron tres ensayos de práctica acerca de cómo elegir una inversión. Cuando todos terminaron de leer las instrucciones y de realizar los ensayos de práctica, iniciaba la sesión. Esta consistió de 45 ensayos, divididos en tres bloques, cada uno de 15 ensayos, separados con un mensaje que anunciaba el inicio de un nuevo juego de bienes públicos, con el fin de dirigir la atención de los participantes al nuevo valor de número de jugadores. Cada individuo fue expuesto a una alta contribución simulada del grupo, manipulado mediante el "fondo común acumulado", que consistió, en promedio, del $80 \%$ del total que podían aportar los sujetos virtuales. La aportación bajaba lo suficiente como para no permitir ganancias en 6 y hasta 7 ensayos, de los 45 programados. La información que se dio sobre esta variable fue la cantidad que se acumuló por inversiones, incluyendo lo que aportó el sujeto focal, y cuanto recibiría cada jugador al final de ese ensayo.

Cada ensayo constaba de cuatro acciones del participante: a) decidir lo que aportaba al "negocio común"; b) presionar el botón "continuar" cuando se le informaba cuántos puntos se acumulaban a su contador personal; c) esperar cerca de 10 segundos mientras se simulaba que se "calculaban intereses de las aportaciones"; y d) presionar el botón "continuar" cuando se le informaba la aportación grupal, las ganancias para todos los jugadores, y los puntos que se acumulaban a su contador personal. El número de jugadores, el punto de provisión y el contador de ganancias estuvieron visibles toda la sesión. La variable dependiente fue la magnitud de inversión individual al "negocio grupal".

Al término de la sesión se daban los resultados de los puntos obtenidos por cada sujeto, para en ese momento premiar monetariamente a los tres participantes que hubieran obtenido los puntajes más altos.

2. Las instrucciones completas están disponibles con los autores 


\section{RESULTADOS}

Para mostrar el efecto principal del número de jugadores en el juego experimental, se obtuvo el promedio de inversión de todos los participantes para cada fase con independencia del orden en que transitaron por las fases, y se grafica con una línea horizontal, en la Figura 1. En ella puede observarse que en general, la inversión fue mayor al $50 \%$ de los puntos disponibles. También se aprecia una mayor inversión para la fase de 6 jugadores, mientras que no hay diferencias entre la inversión para las fases de 8 y 12 jugadores. Un análisis de varianza para medidas repetidas señala que la diferencia entre la fase de menor número de jugadores y las demás fue significativa $\left(F_{2,14}=\right.$ $4.43, p=.018)$.

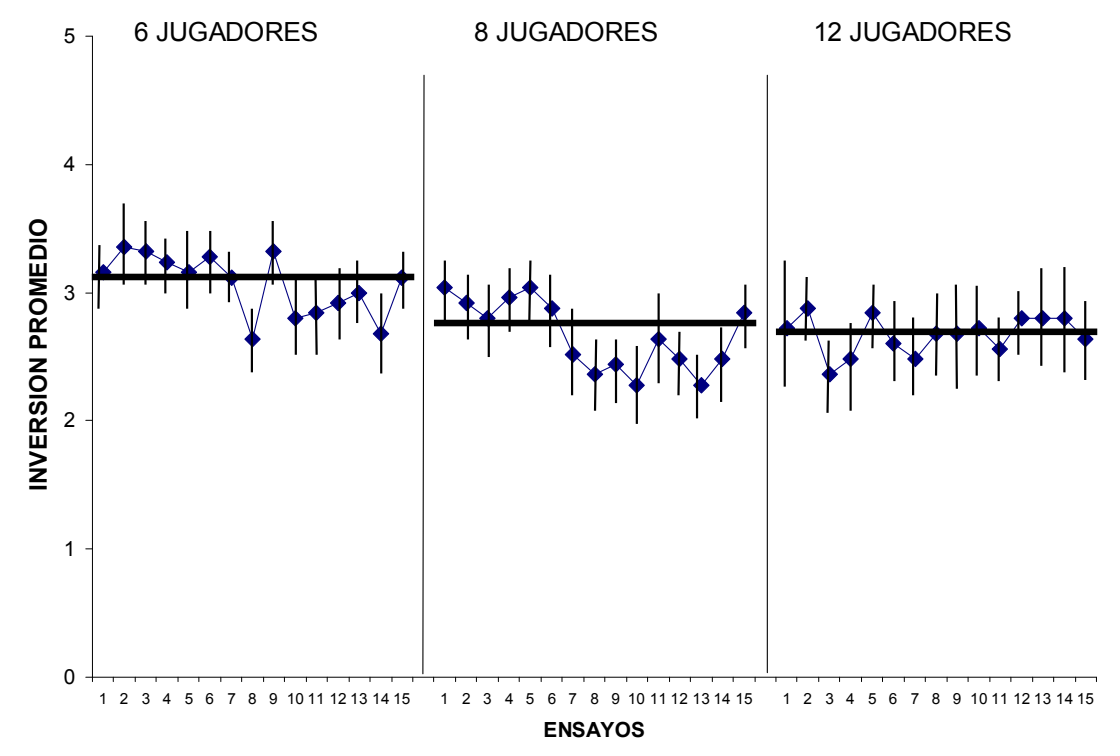

Figura 1. Patrón temporal de la inversión promedio de todos los sujetos, separados por fase de número de jugadores. La línea gruesa indica el promedio por fase, y las líneas delgadas, muestran el error estándar de cada ensayo.

Con el fin de observar el patrón temporal, en dicha Figura 1 se grafica la inversión promedio, durante los quince ensayos de cada fase. Dada la va- 
riabilidad del promedio, en términos de su tendencia, y en términos del error estándar señalado con las líneas verticales, la diferencia entre las fases es menos evidente que con el promedio global, pero aún se nota un mayor nivel de inversión para la fase de 6 jugadores. Un análisis de regresión demuestra que tanto para la fase de 6 jugadores como para la de 8 jugadores, hay una ligera tendencia negativa, que es significativa $(p=0.018$ y $p=.010$, respectivamente). En cambio, en la fase de 12 jugadores la tendencia es positiva, aunque no es significativamente diferente de cero $(p=.437)$.
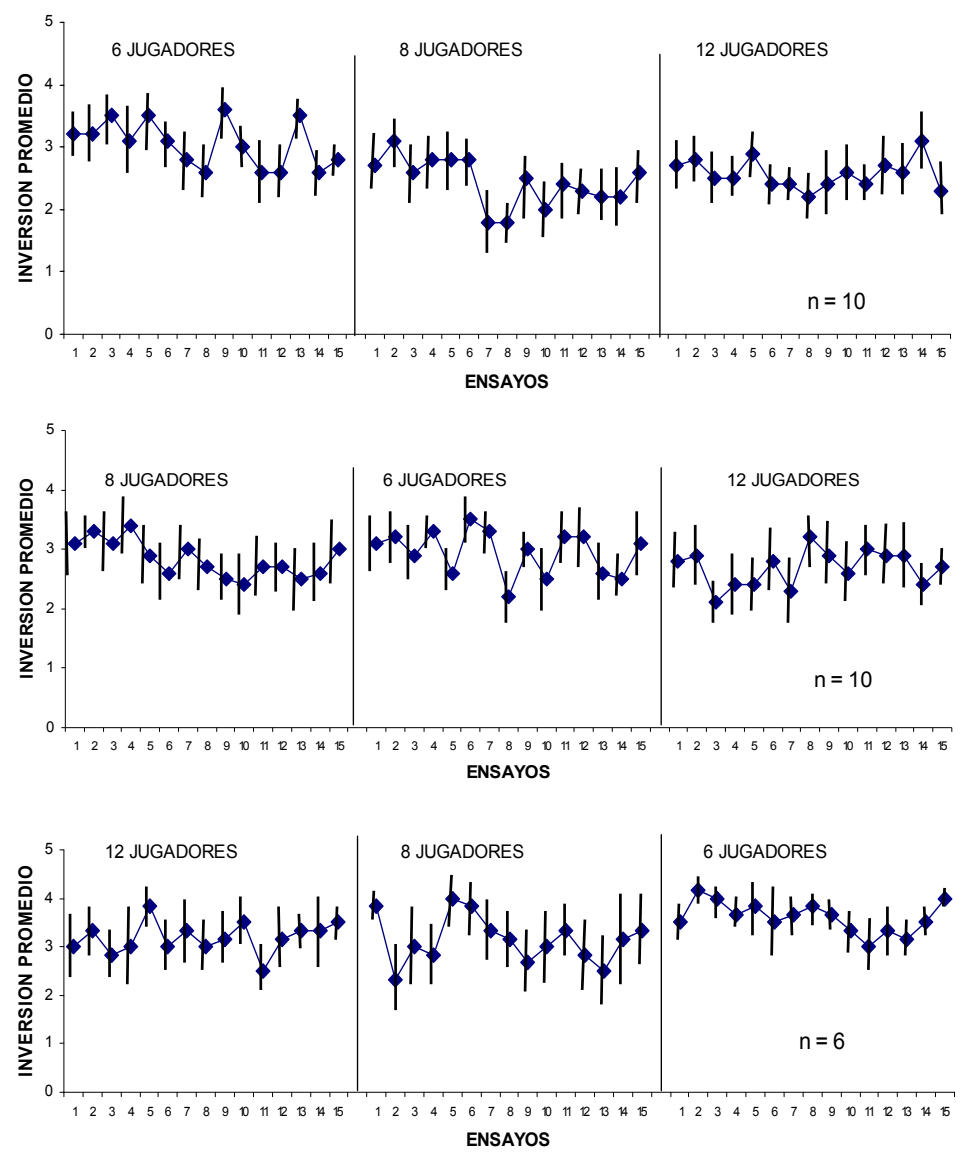

Figura 2. Patrón temporal de la inversión promedio de los sujetos, separados por fases, en tres distintos órdenes de exposición. Las líneas verticales muestran el error estándar de cada ensayo. 
Para determinar si el orden tenía un efecto sobre la inversión, se separaron los individuos que iniciaron con diferente número de jugadores virtuales, como si fueran grupos independientes. La Figura 2 presenta la inversión promedio, para las tres fases, separando a los participantes de acuerdo al orden de la fase inicial. En el panel superior se muestran los datos de los diez participantes que iniciaron con la fase de 6 jugadores. Puede notarse que en la primera fase hubo mayor inversión promedio que en las siguientes dos fases, cuyo nivel oscilaba alrededor del $50 \%$ de las fichas. Las últimas dos fases muestran mayor variabilidad en su tendencia, pero ninguna de las tres muestra una tendencia significativa $(p=.088, p=.056$ y $p=.851$, respectivamente), a pesar de su pendiente ligeramente negativa.

En el panel intermedio de la Figura 2 se presenta la inversión promedio para las tres fases, de los diez participantes que iniciaron con la fase de 8 jugadores. Aquí puede observarse el ligero repunte en la segunda fase, correspondiente a la situación de 6 jugadores. De manera similar las últimas dos fases muestran mayor variabilidad en su pendiente, pero ninguna de las tres muestra una tendencia significativa $(p=.021, p=.314$ y $p=.422$, respectivamente), a pesar de la pendiente ligeramente negativa de la primera.

El panel inferior de la Figura 2 presenta la inversión promedio, para las tres fases, de los 6 participantes que iniciaron con la fase de 12 jugadores. Aquí es más evidente el efecto de contraste para producir mayor inversión promedio en la fase correspondiente a 6 jugadores. La segunda fase muestra mayor variabilidad, con mayores valores extremos, pero con el menor nivel de las tres. La primer fase muestra una tendencia a subir, mientras las otras dos presentan una pendiente ligeramente negativa, pero ninguna de las tres es significativa $(p=.534, p=.533$ y $p=.10$, respectivamente).

Con la finalidad de realizar un análisis de grano fino, se compararon los niveles de inversión y sus tendencias temporales, en las transiciones entre fases. Solo se analizaron tres órdenes específicos, dado que fueron los que contenían al menos seis sujetos. La Figura 3 presenta, en su panel superior, la inversión promedio de seis participantes que siguieron el orden de fases 6 , 8 y 12 jugadores, en los ensayos de transición: los últimos de la fase precedente, y los primeros de la fase consecuente. Las líneas horizontales entre los puntos señalan el nivel promedio, mientras que la tendencia se muestra con una flecha y con el valor de la pendiente. La primera transición, de la fase de 6 a la de 8 jugadores, solo representó un cambio mínimo en el nivel, y la segunda, de la fase de 8 a la de 12 jugadores, sí tuvo un cambio significativo en el nivel $(p=.011)$, señalado con un corchete. Cabe mencionar que dentro de la misma condición de 8 jugadores, la fase intermedia, se dio un cambio de nivel significativo entre los primeros y los últimos ensayos $(p=.025)$, señalado también con corchete. En este caso, los ensayos de la primera transición 

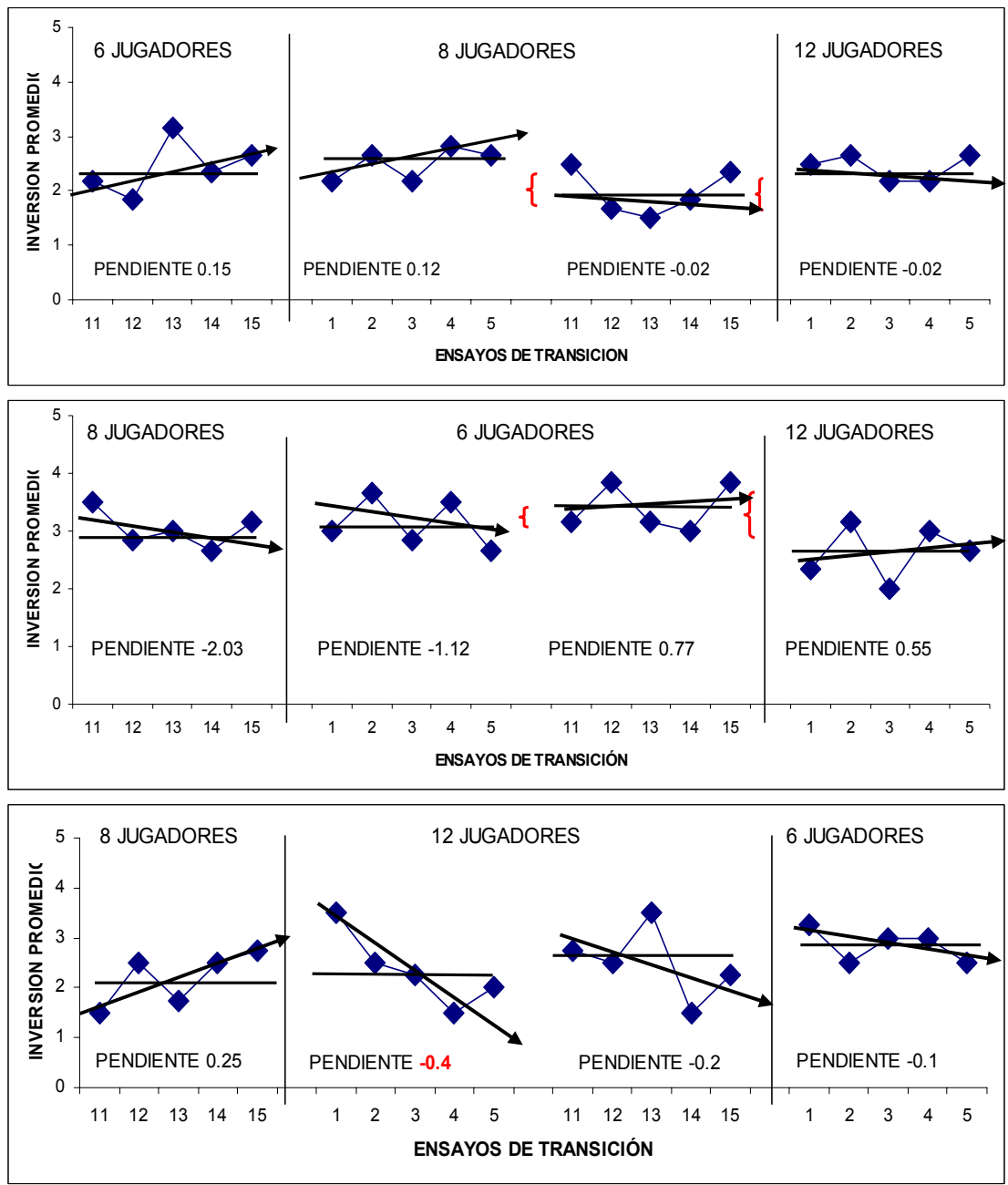

Figura 3. Patrón temporal de la inversión promedio de los participantes con diferentes órdenes de presentación en los ensayos de transición entre fases. Las líneas horizontales señalan el nivel de inversión, la tendencia está indicada por las flechas (se da el valor de la pendiente), y los corchetes señalan cambios significativos de nivel. 
presentaron una pendiente ligeramente positiva mientras que los ensayos de la segunda transición, tuvieron una pendiente casi de cero.

La Figura 3 presenta, en el panel intermedio, la inversión promedio de seis participantes que siguieron el orden de fases 8,6 y 12 jugadores, en los ensayos de transición. La primera transición, de la fase de 8 a la de 6 jugadores, solo representó un cambio mínimo en el nivel, y la segunda, de la fase de 6 a la de 12 jugadores, sí tuvo un cambio significativo en el nivel $(p=.032$, corchete mayor). De igual manera que en el caso del panel anterior, dentro de la misma condición de 8 jugadores, la fase intermedia, se dio un cambio de nivel significativo entre los primeros y los últimos ensayos $(p=.027$, corchete menor). Por el contrario, con estos participantes los ensayos de la primera transición presentaron una pendiente negativa, mientras que los ensayos de la segunda transición, tuvieron una pendiente ligeramente positiva.

El tercer caso de transiciones, con cuatro participantes, se representa en la Figura 3, panel inferior, para el orden 8, 12 y 6 jugadores. En este caso, cada bloque de ensayos tiene un nivel mayor que el precedente. Aun cuando no hay transiciones con cambio significativos en el nivel, la fase de menos jugadores tiene el nivel más alto. Con respecto a la tendencia, la primera transición representó un cambio de una pendiente ligeramente positiva, a una claramente negativa, y la segunda transición no tuvo más que un ligero efecto en la pendiente, sin cambiar su signo.

Los datos individuales son relativamente consistentes, en su mayoría agrupados alrededor de los 2 o 3 puntos en promedio, y con pocos valores extremos, ya sea de cero o de cinco puntos. Si los individuos solo prefirieran dar el $50 \%$ de los puntos, aún se tendría que explicar por qué invierten más de tres puntos en la fase de 6 jugadores, aún en distintos órdenes. Parece ser que la respuesta proviene del punto de provisión, que aunque fijo en un valor durante toda la sesión, cambiaba su porcentaje de acuerdo al número de jugadores. En el caso de 12 jugadores, se requería solo de 2 puntos por sujeto (el $40 \%$ ) para cumplir el punto de provisión. En el caso de 8 jugadores se requería un poco más, el $60 \%$ (tres puntos) para alcanzarlo, pero con solo 6 jugadores, la exigencia subía al 80\% (4 puntos). Para determinar si los participantes realizaban un cálculo de lo que sería necesario invertir con una base de igualdad, se calculó el porcentaje de inversiones que cumplían o no el criterio. En la Figura 4 se muestra un histograma que presenta el porcentaje de inversiones para cada valor de puntos. La flecha señala la barra del valor de inversión que cumplía exactamente el punto de provisión. Solo en el caso de 6 jugadores virtuales, la barra del rango de exactitud es la más alta, con un $54 \%$ de ocurrencia. Para el caso de 8 jugadores, la barra del rango de exactitud está empatada con la siguiente de mayor valor, con un $35 \%$, y para 12 jugadores, la barra del rango de exactitud está en tercer lugar. Es evidente en la gráfica que los participantes prefieren en cualquier caso, invertir de 
más, la mayoría de las veces 4 puntos, pero en forma más acentuada en el caso de 6 jugadores.

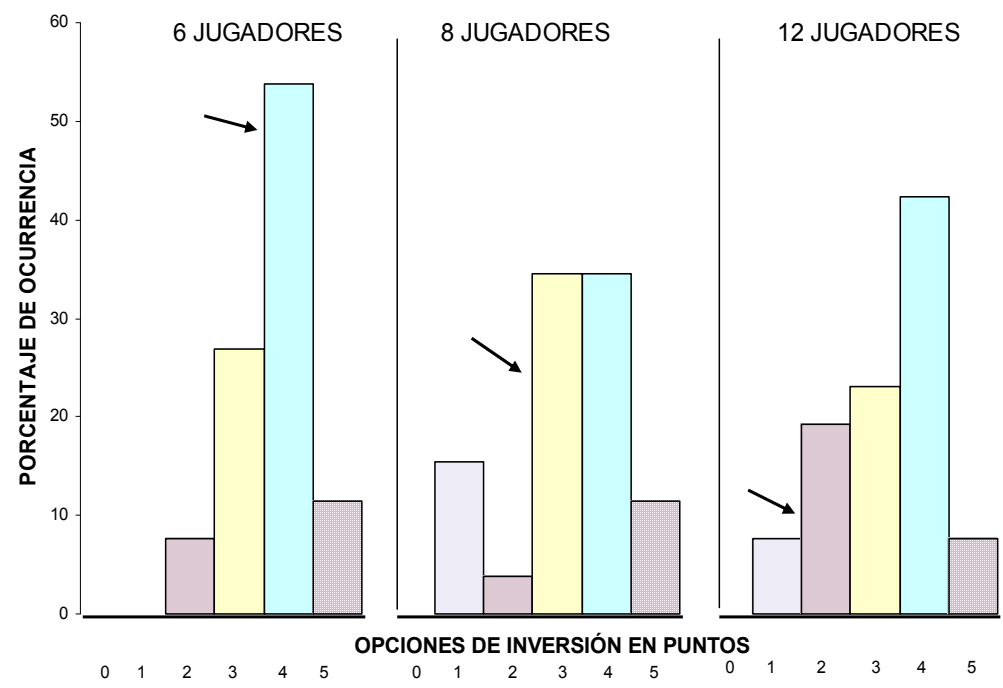

Figura 4. Porcentaje de ocurrencia de las opciones de inversión en puntos de todos los participantes, separados por fase de número de jugadores. Las flechas indican el valor mínimo de inversión requerido.

\section{DISCUSIÓN}

Los resultados confirman que, simulando una alta cooperación del grupo de referencia, los individuos son sensibles al número de jugadores y su relación con el punto de provisión, aportando solo un poco más del mínimo requerido. Esto demuestra que los participantes atienden a estas variables como señales de lo que se esperaría que cada sujeto aportara, y que una cooperación alta del grupo induce altos índices de aportación, en concordancia con la hipótesis de la cooperación condicional (Kesser, 2000, Fischbacher, et al., 2001).

Como en el presente experimento no se permitió la interdependencia entre los individuos, sino que se simuló con jugadores virtuales, se tienen que señalar algunas discrepancias y también algunas coincidencias con los datos 
obtenidos en el campo. La literatura reporta que aunque hay ciertas excepciones, la mayoría de los participantes enfrentados a un dilema de bienes públicos deben decidir entre contribuir todo o nada (Sally, 1995; Ledyard, 1995). En el presente experimento, al permitir un rango de opciones, aun pequeño, se nota mayor variabilidad, y que los jugadores evitan los valores extremos, pocas inversiones en ceros y de igual forma, escasean las inversiones con el total de cinco. Al mantener constante la aportación del grupo virtual, y en un nivel bastante alto, una respuesta de no aportar en absoluto hubiera sido bastante fructífera. Al contrario de lo que se esperaría desde un punto de vista económico racional (Resnik, 1998), ninguno de los 26 participantes desarrolló tal curso de acción.

Cualquiera de estos individuos que hubiera aportado cero, se habría quedado con 5 puntos por ensayo y además recibiría las ganancias de un grupo virtual muy cooperativo. De igual forma, un sujeto con mucha confianza en la cooperación del grupo podría haber incrementado sus ganancias aportando todos los puntos en cada ensayo. La mayoría de los participantes, en promedio, optó por distribuir por igual sus puntos entre la cuenta individual y la grupal. Esto significa contribuir con dos o con tres puntos por ensayo. Con el punto de provisión fijo, para 12 y 8 jugadores, la exigencia mínima por sujeto era de dos y tres puntos, solo para 6 jugadores, la exigencia subía a cuatro puntos por ensayo, por sujeto. Los datos muestran que los participantes fueron sensibles a esta situación.

Si este experimento hubiera comparado tres grupos con diferentes números de jugadores virtuales, después de 15 ensayos se reportarían dos resultados interesantes: En primer lugar, no existiría evidencia del patrón temporal tantas veces encontrado en bienes públicos; contribuciones que inician cerca del $50 \%$ pero que declinan con el tiempo hasta que después de 10 o menos ensayos, prácticamente ya nadie aporta. En el presente estudio, con más ensayos de práctica, la tendencia no presenta cambios sustanciales, manteniéndose estable. Las diferencias en el procedimiento se refieren a que en el caso tradicional, la cooperación del grupo es la variable dependiente, y es dinámica. En este experimento, la cooperación del grupo se controló como una variable que puede confundir el efecto de otras variables, y al mantenerla constante, produjo estabilidad en la aportación, apoyando la idea de que los individuos son más sensibles a esta cooperación condicional, que a la búsqueda egoísta de su único beneficio, según postula la ecuación 1.

En segundo lugar, no se habrían hallado diferencias entre los grupos independientes. Esto llevaría a concluir que la variable número de jugadores no presenta ningún efecto en bienes públicos. Aquí demostramos que la variable número de jugadores tiene su efecto debido a dos razones: la primera por su relación con el punto de provisión. Este punto señala el nivel de aportación que en conjunto producirá ganancias, y si se especifica el número 
de jugadores, se puede calcular cual es el mínimo de aportación individual requerido. La Figura 4 presentó una distribución distinta para cada valor del número de jugadores. La moda de la distribución fue para el mínimo de aportación individual requerido, o un valor mayor. La segunda razón se refiere al efecto de contraste. En este experimento, dada la necesidad de comparar valores distintos del número de jugadores, se usaban varios "juegos”, separados por bloques, que como había demostrado Andreoni (1988), reiniciaba el nivel de aportación de un primer juego. Sin embargo, en el segundo juego se observó un cambio de nivel para los últimos ensayos, y un cambio de nivel en la transición al tercer juego. Si los participantes iniciaban con la fase que exigía mayor aportación individual, el contraste se daba reduciendo ese nivel de aportación, en caso contrario, subiendo dicho nivel. Sin la opción de poder comparar diferentes valores del número de jugadores con el mismo punto de provisión, al mantenerse constante la "cooperación" del grupo virtual, no se requería de un cambio en su estrategia, ya que cualquiera produciría buenas ganancias.

A diferencia de lo que podría suponerse, el número de jugadores no tuvo un efecto directamente proporcional sobre la inversión. Esto apoya el argumento de que los participantes atendieron a esta variable como una señal de cuanto debían aportar si se requería alcanzar el punto de provisión. Este hallazgo es acorde con el de Isaac, Schmitz, y Walker (1989), y en contradicción a los de Komorita y Lapwort (1982), aunque en otros experimentos no se han encontrado diferencias (Isaac y Walker, 1988). Estas contradicciones han llevado a Komorita y Parks (1994) a señalar que "el tamaño del grupo por sí mismo no parece afectar la conducta individual... solo cuando otra cosa cambia junto con el tamaño del grupo" (p. 63). Ahora sabemos que debe cambiar el mínimo de aportación individual requerido para alcanzar el punto de provisión y, además, debe permitirse la comparación intra-sesión.

Por cuestiones prácticas no se probaron aquí valores de número de jugadores más altos, que pudieran tener un efecto, aunque Isaac y Walker (1988) no encontraron diferencias hasta con grupos de 120 individuos, dado que se hacían las comparaciones entre grupos y sin relación con un punto de provisión.

Aunque la hipótesis de cooperación condicional se postuló para datos dinámicos, donde la aportación individual cambia con el paso de los ensayos, debido al cambio de la cooperación grupal, sirve como una primera explicación de los datos aquí obtenidos. En esta simulación de bienes públicos, la cooperación grupal se mantuvo elevada, y la aportación individual se mostró sensible al binomio punto de provisión y número de jugadores. Esto hizo que la búsqueda de patrones como los encontrados por Fischbacher, et al. (2001), se tuviera que hacer de acuerdo con el mínimo que correspondía de inversión, señalado con dicho binomio. De esta manera, aquí se clasifica el 
comportamiento de los individuos como "egoísta", "condicional" o "altruista" cuando invierten menos, igual o más que el mínimo de aportación individual requerido, respectivamente. De la figura 4 se pueden obtener los totales para cada valor del número de jugadores: con seis jugadores tenemos el $34 \%$ de ocurrencia de comportamiento "egoísta", el 54\% de "condicional" y el $11 \%$ de "altruista". Con ocho jugadores los porcentajes cambian a un $19 \%$ de ocurrencia de comportamiento "egoísta", el $8 \%$ de "condicional" y el $46 \%$ de "altruista". Finalmente, con doce jugadores se tiene el $27 \%$, el $23 \%$ y el $50 \%$ de ocurrencia de comportamiento "egoísta", de "condicional" y de "altruista", respectivamente.

Que el procedimiento haya permitido simular diferentes grupos virtuales, tiene al menos dos ventajas. Una consiste en que, al permitir controlar el ambiente social del sujeto, demuestra que aunque hay variabilidad en los niveles individuales de aportación, hay generalidad en los patrones de cambio entre diferentes "grupos", por un efecto de contraste. La otra virtud es que permite apoyar la idea de que la cooperación no es un rasgo del individuo, más bien consiste en el ajuste del comportamiento social ante diferentes restricciones ambientales. En el estudio de Yi y Rachlin (2004) la estrategia de reciprocidad produjo mayor cooperación individual que la cooperación grupal aleatoria, solo si los participantes iniciaban cooperando, de otra manera, se enfrascaban en la trampa de no cooperar, que se castigaba con nula cooperación del grupo. Como su juego consistía en un dilema del prisionero para 5 personas, la matriz de pagos no es directamente comparable con la de bienes públicos. Lo más cercano a patrones individuales que ellos encontraron lo describen como jugadores que "escaparon de la trampa" o jugadores que se "entramparon". En su Experimento 3, lograron "sacar de la trampa" a los participantes que habían caído en ella, al hacer un alto en el juego y reiniciar la reciprocidad para iniciar cooperando, un arreglo muy similar al cambio de fase del presente experimento, que como mostró Andreoni (1988) produce un aumento en la cooperación individual. Aquí solo se añade que el uso de jugadores virtuales permite más posibilidades de estudio sistemático de procesos de interdependencia entre el individuo y un grupo de referencia.

El efecto de la probabilidad de cooperación del grupo se propuso como variable explicativa para el patrón dinámico de aportación en bienes públicos, y cuando se preguntan las preferencias por contribución al bien público por cada contribución promedio de los miembros del grupo (p.e, Fischbacher, Gachter y Fehr, 2001; Santoyo y López, 2003). Sin embargo, los presentes datos sugieren otra posibilidad; en una situación donde las ganancias se comparten entre el grupo, los individuos intentan que los demás respondan en cierta dirección que consideran "adecuada", y que se puede calificar como "justa" o "equitativa". Esto coincide con otros autores que postulan que los individuos tienen preferencias con contenido social (Camerer y Fehr, 2004). 
Estas preferencias se refieren a cómo la gente coloca en rangos diferentes distribuciones de pagos materiales a sí mismos y a otros. Se ha demostrado que la gente puede gastar recursos ya sea para reducir o para incrementar el pago de otros. Esto indica que, además de tomar decisiones que buscan su propio interés, la gente se comporta algunas veces como si tuviera preferencias altruistas, y algunas otras con preferencias por la equidad y la reciprocidad. Fehr y Gachter (2000), mostraron que añadir una segunda fase de "castigo" a un juego de bienes públicos, tenía un efecto que mejora la cooperación. Ellos permitieron en esta segunda fase que cada jugador pudiera asignar castigo de reducción de puntos a cualquiera de los demás jugadores, aunque el castigar también incluía que el castigador perdiera puntos. Como el castigar tenía un costo asociado, nuevamente el auto-interés predeciría que a nadie le convendría ser castigador. En el experimento de Fehr y Gachter (2000), sí hubo jugadores que castigaban, y lo hacían a los egoístas, que en consecuencia, incrementaron su tasa de cooperación. El castigo produjo reciprocidad forzada. Este es otro ejemplo de que los participantes no solo buscan ganancias, sino que son capaces de cargar con pérdidas, si con ello restauran la equidad, o como lo suponemos aquí, ganan control del intercambio.

Finalmente, los presentes resultados van en la dirección de los hallazgos que muestran que las ganancias no son la única motivación en el intercambio social, y que en ocasiones las variables psicológicas deben tener mayor peso en la explicación de la cooperación. Incluso en ciertas condiciones de distribución de consecuencias no compartidas, se ha demostrado que los participantes prefieren las contingencias individuales a las sociales, aunque obtengan menos ganancias (Ribes, Rangel, Casillas, Alvarez, Gudiño, Zaragoza y Hernández, 2003). Los presentes resultados pueden tener implicaciones en situaciones cotidianas, donde la cooperación puede fomentarse con reciprocidad y donde el número de participantes sirve como señal de cuanto aportar.

\section{REFERENCIAS}

Andreoni, J. (1988). Why Free Ride? Journal of Public Economic, 37, 291-304. Axelrod, R. (1984). The Evolution of Cooperation. Nueva York: Basic Books.

Bowles, S. (2004). Microeconomics: Behavior, Institutions and Evolution. New York, Russel Sage.

Brechner, K. C. (1977). An experimental analysis of social traps. Journal of experimental social psychology, 13, 552-564.

Camerer, C. F. y Fehr, E. (2004). Measuring social norms and preferences using experimental games: A guide for social scientists. En J. Henrich; R. Boyd; S. Bowles; C. Camerer; E. Fehr y H. Gintis (eds.) Foundations of Human Sociality: Economic 
Experiments and Ethnographic Evidence from Fifteen Small-Scale Societies. (pp. 55-95) New York: Oxford University Press.

Fehr, E. y Gachter, S. (2000). Cooperation and punishment in Public Goods Experiments. American Economic Review, 90, 980-994.

Fischbacher, U., Gachter, S., y Fehr, E. (2001). Are People Conditionally Cooperative ?- Evidence from Public Goods Experiments. Economics Letters. 71, 397-404.

Isaac, R. M. y Walker, J.M. (1988). Group size effects in public goods provision. Quarterly Journal of Economics, 103, 179-199.

Isaac, R. M., Schmitz, D. y Walker, J. M. (1989). The Assurance Problem in a Laboratory Market. Public Choice, 62, 217-236.

Kesser, C. (2000). Cooperation in Public Goods Experiments. Discussion Paper 11988177, University of Montreal, and CIRANO Scientific Series 2000s-04, Montréal.

Kollock, P. (1998). Social dilemmas: The anatomy of cooperation. Annual Review of Psychology, 46, 183-207.

Komorita, S.S., y Lapworth, C. W. (1982). Alternative choices in social dilemmas. Journal of Conflict Resolution, 26, 692-708.

Komorita, S S., y Parks, C. D. (1994). Social dilemmas. Madison, W I: W C B Brown y Benchmark.

Ledyard, J. (1995). Public Goods: A Survey of Experimental Research. En : A. Roth y J. Kagel, (Eds.). Handbook of Experimental Economics. (pp. 111-194). Princeton: Princeton University Press.

Platt, J. (1973). Social traps. American Psychologist, 28, 641-651.

Resnik, M. D. (1998). Elecciones: una introducción a la teoría de la decisión. Barcelona: Gedisa.

Ribes, E.; Rangel, N.; Casillas, J. R.; Álvarez, A.; Gudiño, M.; Zaragoza, A. y Hernández, H. (2003). Inequidad y Asimetría de las consecuencias en la elección entre contingencias individuales y sociales. Revista Mexicana de Análisis de la conducta, 29, 2, 131-168.

Santoyo, C. (2001). Trampas sociales y negociación: Una perspectiva conductual. En C. Santoyo y F. Vázquez (compiladores). Procesos Psicológicos de la Negociación y la toma de decisiones. (pp. 73-104). México: UNAM.

Santoyo, C. y López, C., E. (2003). Dilemas en Bienes Públicos en Unidades Habitacionales: Una Perspectiva Integrativa. Revista Mexicana de Psicología, 20, 2, 165-175. 